\title{
Nanoparticle Reservoirs for Paper-Only Immunosensors
}

\author{
Alejandra Alba-Patiño, ${ }^{+1,2}$ Cristina Adrover-Jaume, ${ }^{+, 1,2}$ Roberto de la Rica*,1,2 \\ ${ }^{1}$ Multidisciplinary sepsis group, Balearic Islands Health Research Institute (IdISBa), \\ Son Espases University Hospital, S Building, Carretera de Valldemossa 79, 07120 \\ Palma de Mallorca, Spain. \\ ${ }^{2}$ Department of Chemistry, University of the Balearic Islands, Carretera de Valldemossa \\ km 7.5, 07021 Palma de Mallorca, Spain
}

\section{Contents:}

Figures S1-S7 

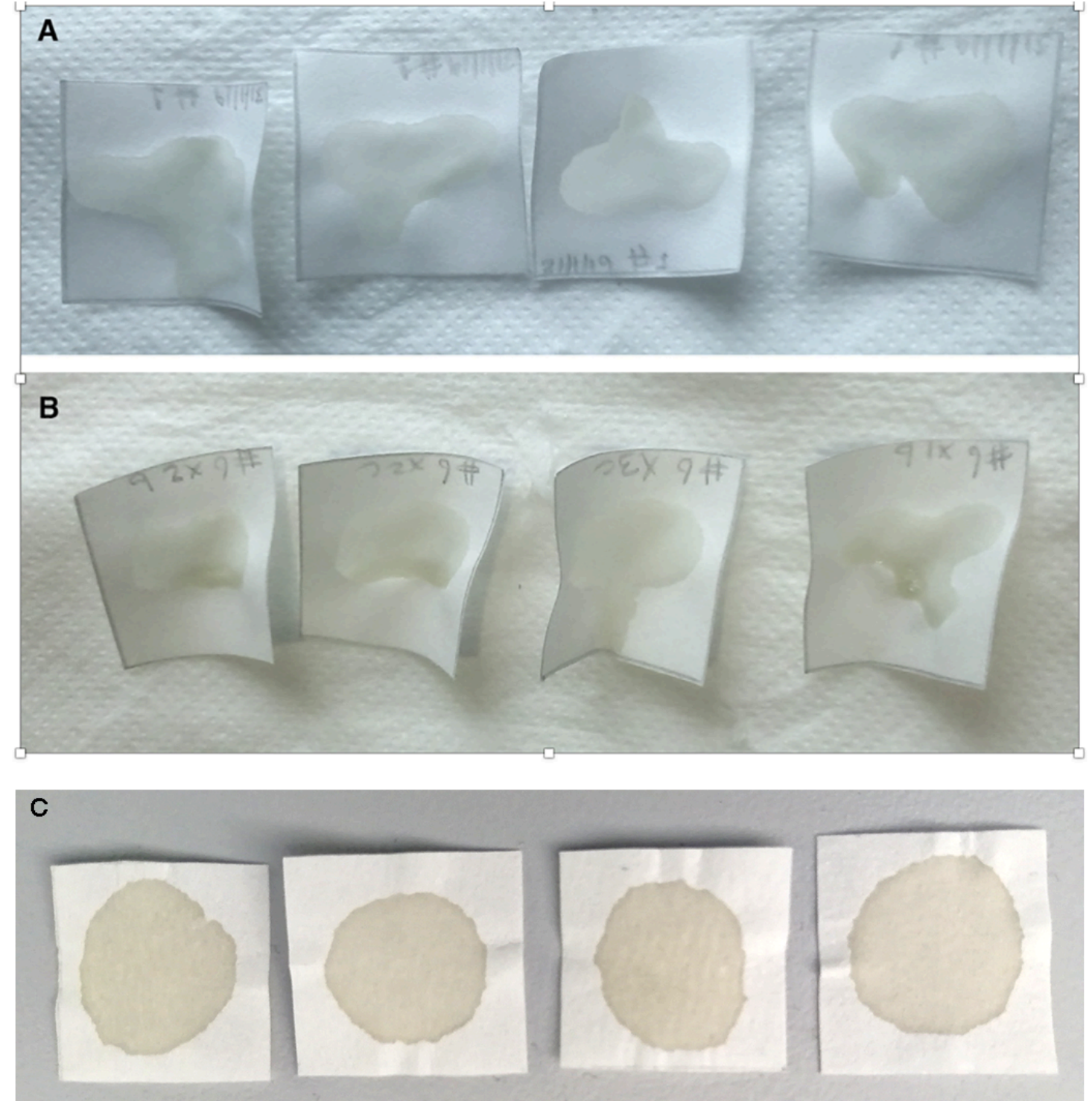

Figure S1. Photographs of filter paper after addition of 30\% PSS; (A) Whatman \#1 (pore size $11 \mu \mathrm{m}$ ); (B) Whatman \# 6 (pore size $3 \mu \mathrm{m})$; (C) Whatman \# 41 (pore size $22 \mu \mathrm{m}$ ). It took a long time for the PSS solution to be adsorbed by the filter paper for paper types 1 and 6 , and the substrates became heavily warped upon drying. 


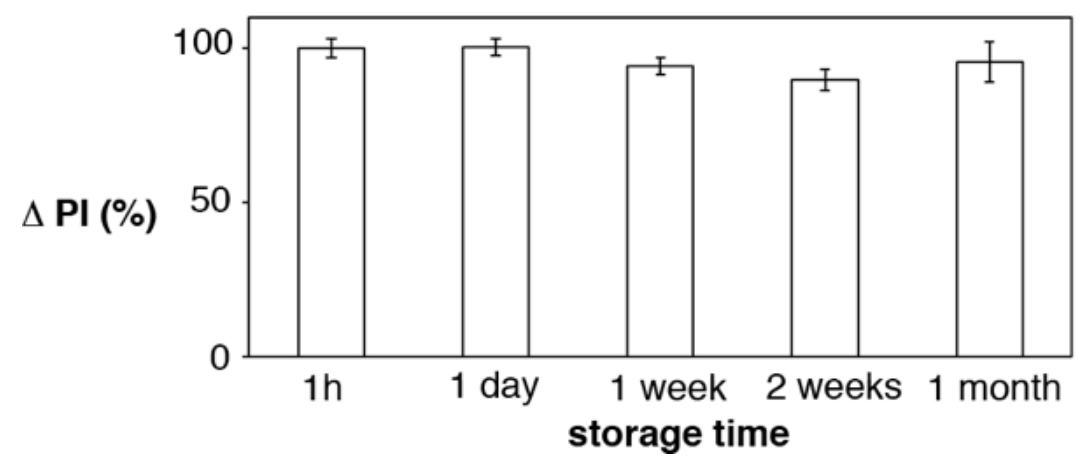

Figure S2. Variation of the colorimetric signal (pixel intensity, PI) resulting from biospecific avidin-biotin interactions when the assay is performed using dry nanoparticle reservoirs stored for different times at room temperature. The percentage signal was calculated with respect to the reservoirs stored for $1 \mathrm{~h}$. Error bars are the standard deviation $(\mathrm{n}=3)$. Student's t-test shows that mean values at different times are not statistically different from the mean value at $t=1 \mathrm{~h}(\mathrm{p}>0.05$ in all cases). 

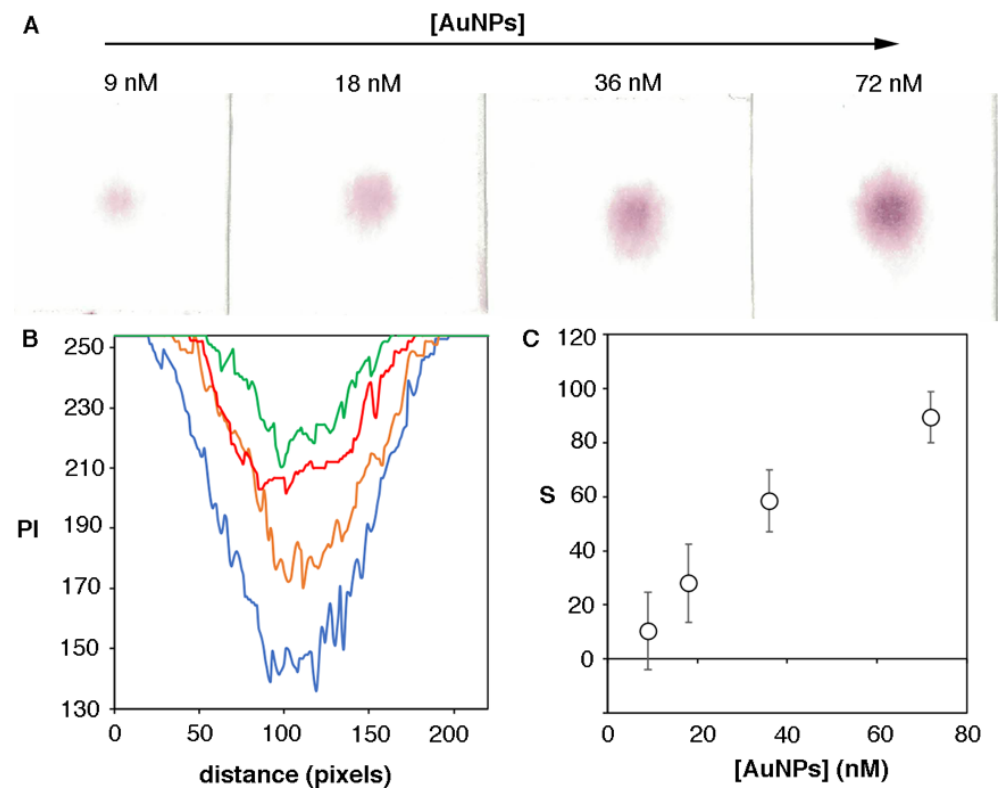

Figure S3. Test for the formation of a "coffee ring" made of biotinylated BSA. To rule out that biotin-BSA generates a "coffee ring" upon drying, paper substrates containing dry biotinylated BSA were modified with a drop of avidin-decorated nanoparticles $(2 \mu \mathrm{L})$ at different concentrations instead of transferring them from the nanoparticle reservoir. In Figure S3A the colored spots obtained after washing the nanoparticles 3 times with PBST do not show a coffee a ring. Actually, the colored spots are more intense in the center, since the nanoparticles diffuse laterally to a larger extent in the absence of PSS. Profile analyses in Figure S3B corroborate that no coffee rings are formed when the nanoparticles are dispensed as a drop rather than by transferring them from the reservoir. Figure S3C shows the quantification of the colorimetric signal in 3 independent experiments (error bars are the standard deviation). 

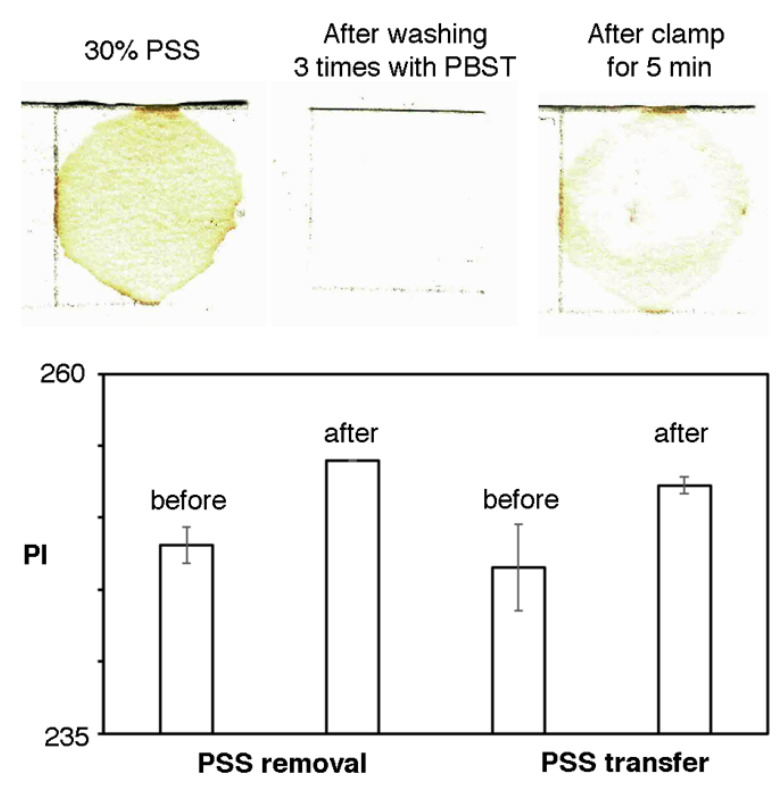

Figure S4. The characteristic yellow color of PSS was used to follow its elimination after a washing procedure ( 3 times with $1 \mathrm{~mL}$ of PBST) or after 5 min in contact with a wet substrate (transfer). Error bars are the standard deviation $(n=3)$. As shown above, the washing procedure completely eliminated the yellow color from the paper, with the subsequent increase in pixel intensity. The transferring process also removed the color from the center of the paper, where the clamp pressed it against the receiving substrate. These experiments show that PSS is transferred along with the nanoparticles upon addition of liquids or after pressing it against a wet receiving substrate. Error bars are the standard deviation $(\mathrm{n}=3)$. 

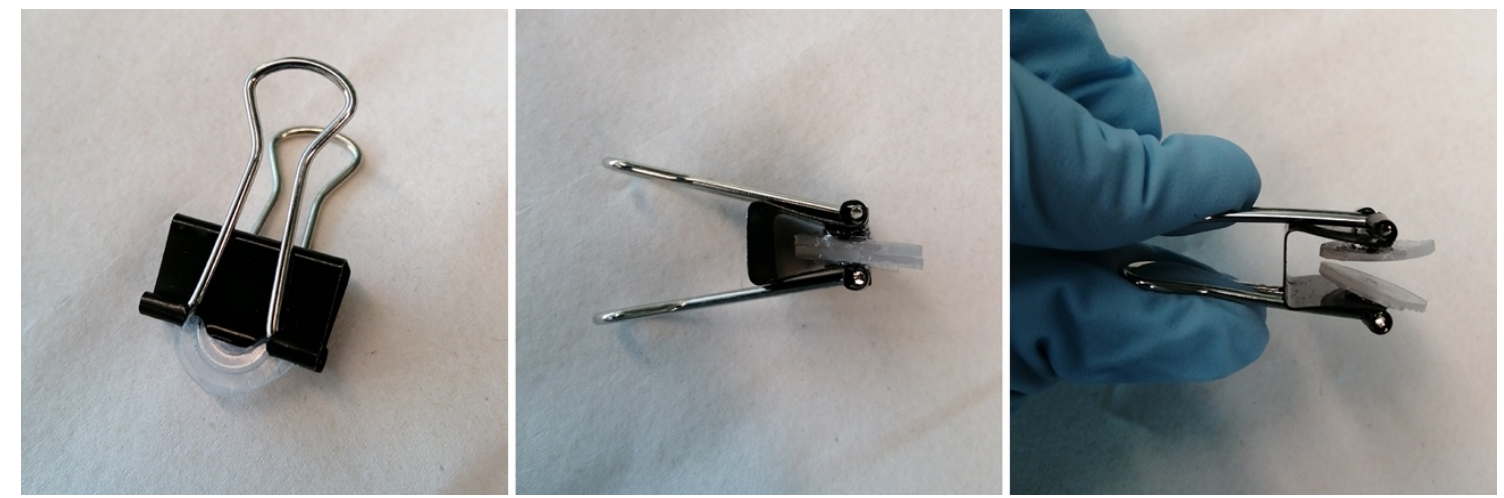

Figure S5. Pictures of the clamp used to transfer nanoparticles in Figure 7. It consists of a paper clamp that has been modified with 2 flat objects in order to apply pressure evenly in the center of the paper biosensor. The flat objects are two Eppendorf tube caps that have been glued to the clamp. 


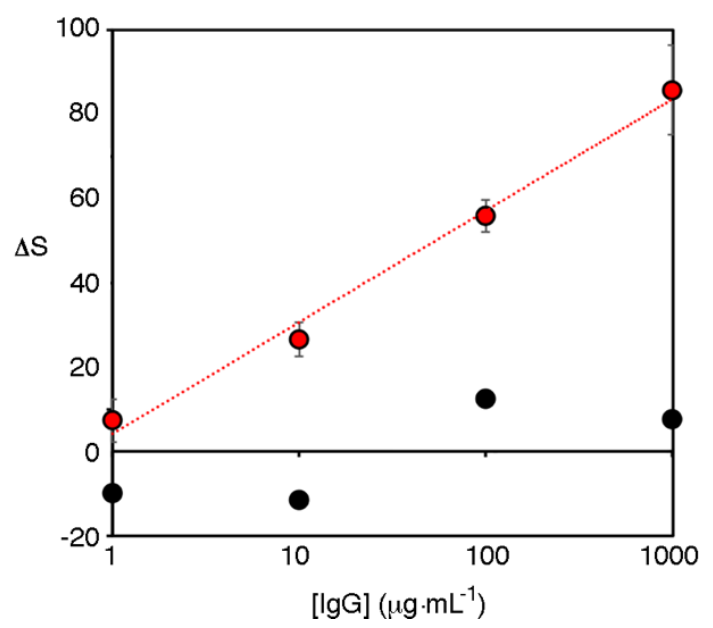

Figure S6. Detection of mouse IgG with citrate-capped nanoparticles. Nanoparticles were modified with antibodies with the following procedure based on a previously published protocol (Lab Chip, 2013,13, 386-390). First the $\mathrm{pH}$ of the nanoparticle dispersion was adjusted to 9 with sodium bicarbonate $(10 \mathrm{mM}, \mathrm{pH} 11)$. Then anti-mouse IgG Fc specific $(100 \mu \mathrm{L}, 1 \mathrm{mg} / \mathrm{mL})$ or anti-E. coli $(100 \mu \mathrm{L}, 1 \mathrm{mg} / \mathrm{mL})$ was added to the nanoparticles under agitation $(1.5 \mathrm{~mL}, 650 \mathrm{rpm})$ for $20 \mathrm{~min}$. Subsequently BSA was added to stabilize the colloids $(100 \mu \mathrm{L}, 1 \mathrm{mg} / \mathrm{mL})$ for $20 \mathrm{~min}$. The nanoparticles were then centrifuged at $7000 \mathrm{rpm}$ for $6 \mathrm{~min}$ and resuspended in $6 \mu \mathrm{L}$ of PBS-BSA. Nanoparticle reservoirs were prepared by adding $1 \mu \mathrm{L}$ of nanoparticles to a piece of paper modified with $30 \%$ PSS. To prove that the nanoparticles can be transferred and that they retain the ability to specifically recognize their antigen, a receiving paper substrate was modified with mouse IgG $(5 \mu \mathrm{L} ; 1000,100,10,1$ or $0 \mu \mathrm{g} / \mathrm{mL})$. After drying and blocking with PBSA-BSA $(1 \mathrm{~mL})$ the reservoir was pressed against the receiving paper with the aid of the clamp shown in Figure S5. Then the biosensors were washed 3 times with PBST and the images were scanned. The colorimetric signal was calculated as the increase in pixel intensity with respect to the blank experiment with no mouse $\operatorname{IgG}(\Delta \mathrm{S})$. In Figure S6 nanoparticles modified with anti-mouse IgG show a concentration-dependent signal (red dots) that is higher than in control experiments with anti-E. coli (black dots). This demonstrates that citrate-capped gold nanoparticles modified with antibodies can be stored in the proposed paper reservoirs. Error bars are the standard deviation of 3 independent experiments. 


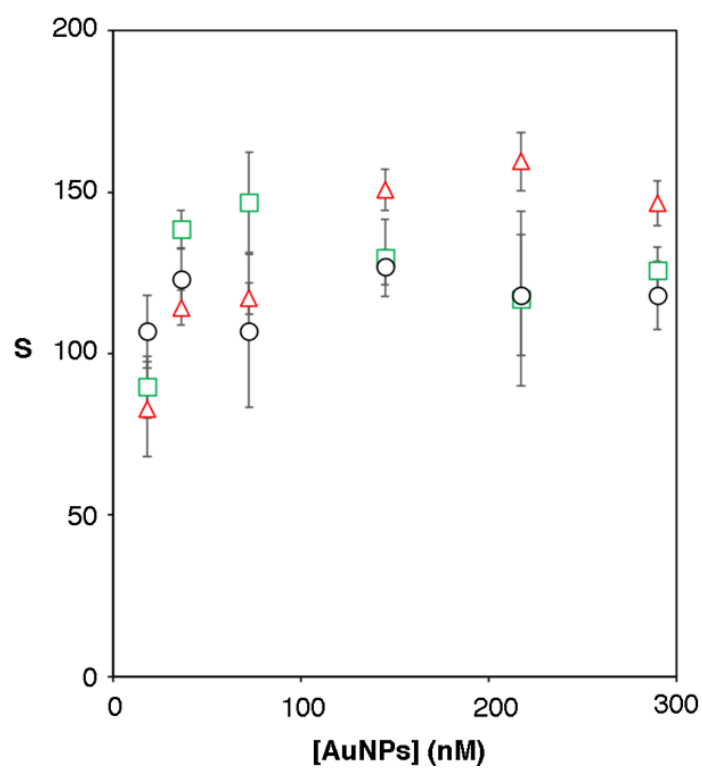

Figure S7. Specific signal obtained from transfer experiments in Figure 6. The signal S was obtained by subtracting the signal of control experiments performed with nonbiotinylated BSA. Error bars are the standard deviation of 3 independent experiments, and therefore indicate a 90\% confidence level. In this Figure, the specific signal was the highest when the receiving paper was Whatman paper \#41 (red triangles) and the nanoparticles were stored in the reservoir with a concentration between 140 and $290 \mathrm{nM}$. The highest specific signal when nanoparticles were stored at a concentration of 36 or 72 nM was achieved with Whatman paper \# 1 (green squares). Specific signals obtained with Whatman paper \#6 are lower, or not significantly different from those obtained with other paper types (black circles, $\mathrm{p}>0.05$ ). 